\title{
Very metal poor Classical Cepheids: variables in IZw18
}

\author{
G. Fiorentino $^{1}$, M. Marconi ${ }^{2}$, G. Clementini ${ }^{1}$, I, Musella ${ }^{2}$, A. Aloisi ${ }^{3}$, \\ F. Annibali ${ }^{3}$, R. A. Contreras ${ }^{1}$ and M. Tosi ${ }^{1}$ \\ ${ }^{1}$ INAF-OABo, via Ranzani 1, 40137, Bologna, Italia \\ email: giuliana.fiorentino@oabo.inaf.it \\ ${ }^{2}$ INAF-OAC, via Moiariello 16, 80131, Napoli, Italia \\ ${ }^{3}$ Space Telescope Science Institute, 3700 San Martin Drive, Baltimore, MD 21218
}

\begin{abstract}
In the framework of an ongoing ACS@HST project (HST program \#10586, PI: Aloisi) we have obtained deep multi-color imaging of the very metal-poor Blue Compact Dwarf galaxy IZw18. The data were acquired in time-series fashion to allow the identification of Classical Cepheids (CCs). The main aim of this project is to constrain both the distance and the Star Formation History of the galaxy. However, as a byproduct these data also provide new insights into the properties of CCs at very low metallicities. We have identified 24 candidate CCs in IZw18. New theoretical pulsation models of CCs specifically for the low metallicity of this primordial galaxy $(\mathrm{Z}=0.0004, \mathrm{Y}=0.24)$ have been computed to interpret our results.
\end{abstract}

Keywords. stars: variables: Cepheids

\section{Introduction}

The Blue Compact Dwarf galaxy IZw18 is one of the most intriguing objects in the Local Universe. It has the lowest nebular metallicity of all known star forming galaxies $\mathrm{Z}=1 / 30-1 / 50 \mathrm{Z}_{\odot}$ (depending on the metallicity scale), and it has long been regarded as a possible example of a galaxy undergoing its first burst of star formation, hence a local analog of primordial galaxies in the distant Universe. Previous HST observations with WFPC2 and NICMOS (Aloisi et al., 1999, Ostlin et al., 2000) detected AGB stars tracing an underlying population about 0.5 Gyr old. However, Izotov \& Thuan, (2004, IT04) failed to reveal the galaxy RGB tip on their ACS@HST data, thus suggesting that IZw18 does not host an old population. Since current estimates for the distance of IZw18 are very uncertain, it may be possible that IZw18 RGB tip was not detected because the galaxy is farther away than previously thought. We have used our ACS@HST deep time-series data of IZw18, to measure the galaxy distance with the CCs.

\section{Data reductions \& Theoretical models}

The proprietary ACS@HST data were combined with IT04 images, reaching a total time baseline of about 2.5 years. Photometric reductions were performed using the ALLFRAME package (Stetson 1987, 1992). Variable stars were detected using both the Optimal Image Subtraction Technique (ISIS 2.2, Alard 2000) and the Welch \& Stetson (1993) variability index. We have identified 24 variable star candidates (triangles in panel a), Fig. 1). Analysis of the light curves is in progress. The $V, I$ light curves of a $\mathrm{CC}$ with period of $8.8 \mathrm{~d}$ (star \#4306) are shown in Fig. 1 (panel b).

New theoretical nonlinear pulsation models of CCs at the metallicity of IZw18 (Z=0.0004, $\mathrm{Y}=0.24$ ) have been computed, (see Marconi et al. 2005 for details on the pulsation code) 

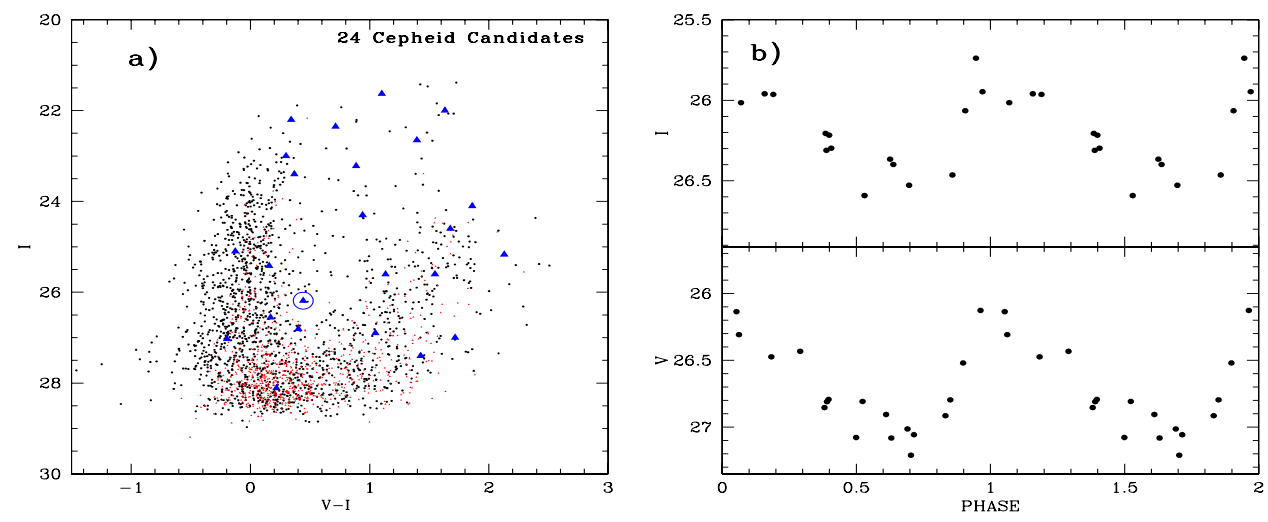

Figure 1. Panel a): CMD of IZw18 obtained combining F555W, F606W and F814W ACS data from IT04 and from Aloisi et al. HST program \#10586. Candidate variables are marked by filled triangles. Panel b): $V, I$ light curves for a $\mathrm{P}=8.8 \mathrm{~d}$ CC (see open circles in panel a).

to constrain the metallicity dependence of the Cepheid Period-Luminosity (PL) relation in the low metallicity regime. The effect of different assumptions in the mixing length parameter $(\mathrm{l} / \mathrm{HP}=1.5-2)$ on the classical pulsation relations ( $\mathrm{PLC}$, Wesenheit and $\mathrm{PL}$ ) and on the theoretical light curves has been investigated. At this low metallicity, the effect of increasing the convection efficiency is negligible on the light curve amplitude, but causes a decrease in effective temperature width of the Instability Strip. The metallicity effect on the PL relations is negligible in this metal-poor range.

\section{Conclusions}

The distance modulus of IZw18 was determined by applying both the theoretical and the empirical approach to our most reliable CCs (namely stars: \#8431 and 4306): 1) Our theoretical Wesenheit relation (Fiorentino et al. 2007, in preparation) gives : $\mu_{0}=31.28 \pm$ 0.26 mag; 2) Ogle's empirical PL-relation for the LMC (Udalski et al. 1999): gives $\mu_{0}=$ $30.71 \pm 0.29 \mathrm{mag}$, for an assumed distance modulus for the LMC of $18.5 \mathrm{mag}$. If we take into account the metallicity correction, we find: 1) a decrease of the distance modulus to: $\mu_{0}=30.40 \pm 0.29 \mathrm{mag}$ if the empirical correction by Kennicut et al. (1998) is adopted; 2 ) an increase of the distance modulus to $\mu_{0}=31.01 \pm 0.29 \mathrm{mag}$, if we extrapolate to the metallicity of IZw18 the theoretical correction by Fiorentino et al. (2002).

\section{References}

Aloisi, A., Tosi, M. \& Greggio, L., 1999, AJ, 118, 302A

Fiorentino, G., Caputo, F., Marconi, M., \& Musella, I., 2002, ApJ, 576, 402

Kennicutt, R. C., Jr., et al. 1998, ApJ, 498, 181

Izotov, Y. I. \& Thuan, T. X., 2004, ApJ, 616, 768I

Marconi, M., Musella, I., \& Fiorentino, G., 2005, ApJ, 632, 590M

Ostlin, G., 2000, ApJ, 535L, 99 O

Welch, D. L. \& Stetson, P. B., 1993, AJ, 105, 1813W

Stetson, Peter B., 1987, PASP, 99, 191S

Stetson, Peter B., 1992, ASPC, 25, 297S

Udalski, A., et al. 1999, Acta Astron., 49, 223 